Provided for non-commercial research and education use. Not for reproduction, distribution or commercial use.

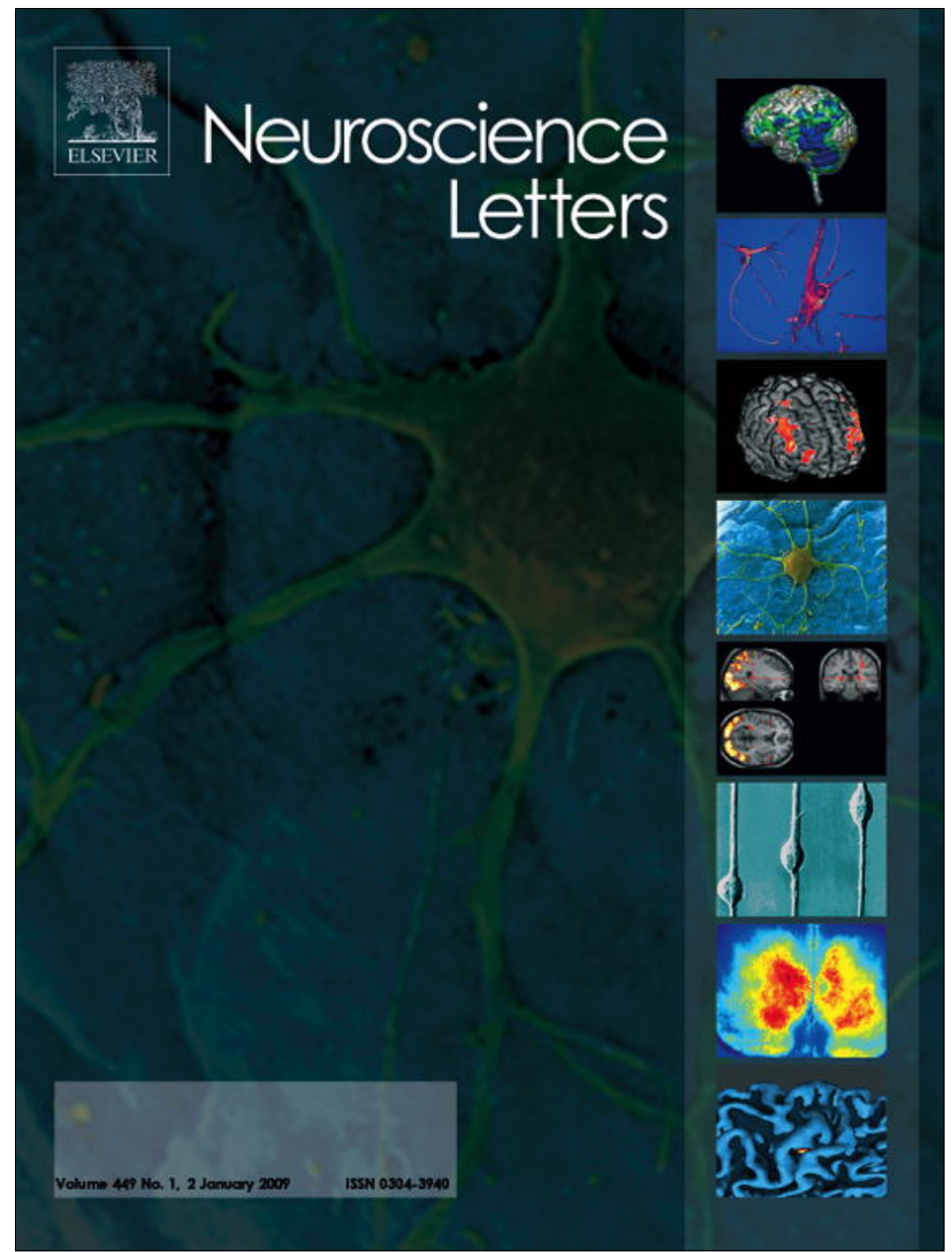

This article appeared in a journal published by Elsevier. The attached copy is furnished to the author for internal non-commercial research and education use, including for instruction at the authors institution and sharing with colleagues.

Other uses, including reproduction and distribution, or selling or licensing copies, or posting to personal, institutional or third party websites are prohibited.

In most cases authors are permitted to post their version of the article (e.g. in Word or Tex form) to their personal website or institutional repository. Authors requiring further information regarding Elsevier's archiving and manuscript policies are encouraged to visit:

http://www.elsevier.com/copyright 


\title{
An fMRI study of acupuncture using independent component analysis
}

\author{
Yi Zhang a, Wei Qin a , Peng Liu ${ }^{\mathrm{b}}$, Jie Tian ${ }^{\mathrm{a}, \mathrm{c}, *}$, Jimin Liang a ${ }^{\mathrm{a}}$, Karen M. von Deneen ${ }^{\mathrm{d}}$, Yijun Liu ${ }^{\mathrm{d}}$ \\ a Life Science Research Center, School of Electronic Engineering, Xidian University, Xi'an, Shaanxi 710071, China \\ ${ }^{\mathrm{b}}$ School of Sino-Dutch Biomedical and Information Engineering, Northeastern University, Shenyang, Liaoning 110004, China \\ c Medical Image Processing Group, Key Laboratory of Complex System and Intelligence Science, Institute of Automation, Chinese Academy of Sciences, \\ Graduate School of the Chinese Academy of Sciences, P.O. Box 2728, Beijing 100190, China \\ ${ }^{\mathrm{d}}$ Department of Psychiatry and Neuroscience, McKnight Brain Institute, University of Florida, 100 Newell Drive, P.O. Box 100256, Gainesville, FL 32610, USA
}

\section{A R T I C L E I N F O}

\section{Article history:}

Received 19 July 2008

Received in revised form 14 October 2008

Accepted 15 October 2008

\section{Keywords:}

Acupuncture

Independent component analysis (ICA)

Functional brain network

fMRI

\begin{abstract}
A B S T R A C T
In this paper, we studied the brain functional networks corresponding to the traditional multiple-block acupuncture task paradigm. Due to the complexity and sustainability seen during acupuncture, we wanted to investigate whether or not the effects during acupuncture are changing according to the multipleblock paradigm. We introduced the data driven method of independent component analysis (ICA) to identify brain functional networks activated during the course of acupuncture and to isolate different networks likely related to different aspects of the acupuncture experience. The comparisons between different resting states disclosed the discrepancies between the pre- and post-needling effects in the brain. Furthermore, the distinction between needle stimulation and the resting state indicated that there existed different functional brain networks. These results also portray time variability during the course of acupuncture.
\end{abstract}

(c) 2008 Elsevier Ireland Ltd. All rights reserved.
Acupuncture originated in ancient China as an alternative and complementary therapeutic intervention. It is gaining popularity in the Western world $[11,13,14]$. The development of imaging techniques, such as positron resonance imaging (PET) and functional magnetic resonance imaging (fMRI), have opened a 'window' into the brain that allows us to obtain an appreciation of the anatomy and physiological function involved during acupuncture in humans and animals non-invasively.

According to Traditional Chinese Medicine (TCM), acupuncture at specific acupoints is able to treat certain types of diseases. Cho et al. [7] reported that acupuncture at vision-related acupoints in the foot, which are used to treat eye diseases in TCM, are activated the visual cortex bilaterally. Zhang et al. [31] demonstrated that electroacupuncture stimulation (EAS) at two pairs of acupoints in the same spinal segment evokes specific responses in brain images obtained with fMRI. The studies of Hui et al. and Wu et al. $[20,28,29]$ on acupoint ST 36 showed significant modulatory effects on the limbic system, paralimbic and subcortical gray structures, while control stimulation showed strong activation mainly at the somatosensory cortex. Kong et al. [21] studied activa-

* Corresponding author at: Medical Image Processing Group, Key Laboratory of Complex System and Intelligence Science, Institute of Automation, Chinese Academy of Sciences, Graduate School of the Chinese Academy of Sciences, P.O. Box 2728, Beijing 100190, China. Tel.: +86 1062527995 ; fax: +86 1062527995.

E-mail address: tian@ieee.org (J. Tian). tion patterns evoked by manual and electroacupuncture, showing that electroacupuncture mainly produced fMRI signal increases in the precentral and postcentral gyri, inferior parietal lobule (IPL), and putamen/insula. Meanwhile, manual needle manipulation produced prominent decreases in fMRI signals in the posterior cingulated cortex (PCC), superior temporal gyrus (STG), and putamen/insula. Yan et al. [30] studied the effects of two different acupoints Liv3 and LI4 versus their sham points, which revealed that acupuncture at acupoints induces specific patterns of brain activity.

These previous acupuncture studies [7,31,20,28,29,21,30] were mainly based on multiple-block design, and fMRI data were analyzed using the whole block paradigm. However, from our analysis, we found that the 'on-state,' during which needle manipulation was performed, consisted of three main components: mechanical stimulation due to needle manipulation, subject expectation of what they would experience and the actual acupuncture effects. Meanwhile, during the 'off-state,' in which manipulation ceases but the needle is still in, expectation and acupuncture effects still remained. There are still two effects during the 'off-state' as compared to the resting state before needle stimulation (RSBNS). Thus, we want to investigate whether or not the 'off-state' and the RSBNS share the same state level and brain functional networks. There also exist different components between the 'on-state' and 'offstate,' so we propose that there exist different functional brain networks within the two states and they should be analyzed separately. 


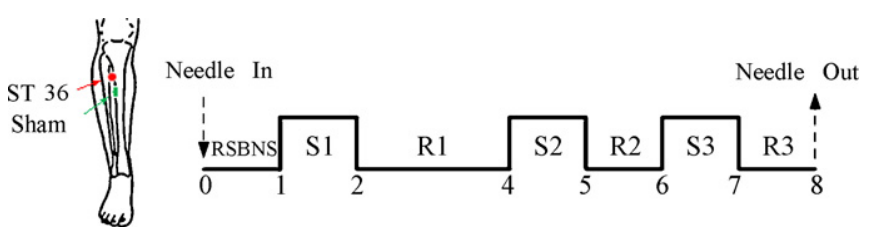

Fig. 1. Anatomical locations of the stimulation point ST 36, nearby sham point and experimental paradigm.

Despite the effects existing during the 'on-state' and 'off-state,' the artifacts are secondary to subtle movements [17], machine noise [27], and cardiac and respiratory pulsations [4], which make up the bulk of variability in the measured fMRI signals. Therefore, we introduce the data driven method independent component analysis (ICA), which is being increasingly applied to fMRI data $[3,23]$. ICA has several advantages as compared to other fMRI analysis methods including correlation and statistical parametric mapping (SPM) [16], in which the time courses and/or spatial extents of anticipated effects must be modeled explicitly prior to analysis. More recently, ICA was adapted to detect the entire default-mode network [29] rather than using an ROI-based approach. ICA is a statistical technique that separates a set of signals into independent-uncorrelated and non-Gaussian-spatiotemporal components [2]. ICA allows not only the removal of high- and lowfrequency artifacts [22], but also the isolation of task-related neural networks $[22,5,6,12]$. In this study, we used ICA to determine the three-dimensional brain topographies and time courses of activations associated with spatially independent components that add up to the measured fMRI signals recorded during acupuncture. Our results suggest that ICA can be used effectively to extract functional networks based on acupuncture stimulation from the remaining components.

The study was performed on 16 right-handed volunteers (8 males and 8 females), aged $24.2 \pm 2.9$ years. All subjects were acupuncture naïve and gave written informed consent as approved by the local Ethics Committee of the Chinese Academy of Sciences. All subjects were in accordance with the declaration of Helsinki.

There were two runs in our experiment. In run 1, real acupuncture was performed at point ST 36 for $8 \mathrm{~min}$. The needle was kept in the point for $1 \mathrm{~min}$ before manipulation, followed by three separate stimulation blocks S1, S2 and S3 as shown in Fig. 1. In run 2 , stimulation was at a non-meridian focus near ST 36 (approximately $2-3 \mathrm{~cm}$ distal lateral). Manipulation and needle depth were identical to run 1 . The two runs were performed every other day, $24 \mathrm{~h}$ apart. In this study we only analyzed the fMRI data from run 1. Because sham is the control, which is predominantly activated in the somatosensory cortex $[20,28,29,30]$, thus it is not possible for it to replicate the specific brain activity seen in acupoint stimulation.

The experiments were carried out in a 3T GE scanner. A gradient echo $\mathrm{T} 2 *$-weighted sequence with in-plane resolution of $3.75 \mathrm{~mm} \times 3.75 \mathrm{~mm}$ (TE $30 \mathrm{~ms}$, TR $1.5 \mathrm{~s}$, matrix $64 \times 64$, FOV $240 \mathrm{~mm}$, flip angle $90^{\circ}$ ) and a set of T1-weighted high-resolution structural images (TE $3.39 \mathrm{~ms}$, TR $2.7 \mathrm{~s}$, matrix $256 \times 256$, FOV $256 \mathrm{~mm}$, flip angle $7^{\circ}$, in plane resolution $1 \mathrm{~mm} \times 1 \mathrm{~mm}$, slice thickness $1 \mathrm{~mm}$ ) were acquired.

Data were preprocessed and analyzed using Statistical Parametric Mapping 5 (SPM5, http://www.fil.ion.ucl.ac.uk/spm). Images were corrected for movement using least square minimization without higher-order corrections for spin history (one subject was excluded because head movement was greater than $3 \mathrm{~mm}$ ), and normalized [15] to the Montreal Neurological Institute (MNI) template. Images were then resampled every $3 \mathrm{~mm}$ using sinc interpolation and smoothed with a $6 \mathrm{~mm}$ Gaussian kernel to decrease spatial noise.
The smoothed data were arranged into Group ICA of the fMRI Toolbox (GIFT, http://icatb.sourceforge.net/). Using the Informax ICA algorithm, the number of independent components (ICs) separated by Group ICA was estimated to be 30 based on Akaike's information criterion (AIC). The mean ICs of all the subjects, the corresponding mean time courses and ICs for each subject were obtained from Group ICA separation and back-reconstruction [5]. We then identified the task-related IC via a close correlation between corresponding mean time course over all the subjects and the task block time course. We obtained the two most critical components separately $(|z|>2)[22,5,6,12]$. One component was obviously attributed to artifacts or noise. Therefore, another one was chosen as the component to represent the effect according to the task paradigm. A one-sample $t$-test (FDR, $P=0.05$, extent threshold $k=20$ voxels) was used to examine the group results for the identified IC of acupuncture.

This identified IC interacted as one functional network during acupuncture stimulation. The activated brain areas detected in the IC were defined as a template corresponding to the task paradigm. We also decomposed the five time periods: the RSBNS state, the first (S1) and second (S2) stimulation blocks, and the first (R1) and second (R2) rest periods separately using Group ICA. The number of ICs was estimated to be 20 . The template was then used to select the 'best-fit' component of the five time periods above [19]. Simple $t$-tests $(P<0.005)$ were used to examine the group results for the identified IC related to each of the different periods.

The functional network of the RSBNS state (Fig. 2) is located in the bilateral frontal gyri (Brodmann areas (BA): BA 9/10/11), bilateral temporal gyri (BA 22/37), inferior and superior parietal lobules (BA 7/40), lateral pre- and postcentral gyri, the anterior cingulate cortex (ACC) (BA 24), parahippocampus (PHIPP), hippocampus (HIPP), lateral insula (BA 13), bilateral thalamus, precuneus (BA 7), caudate, culmen, declive and cuneus.

The S1 stimulation time period evoked network (Fig. 2) includes the bilateral frontal gyri (BA 10/11), lateral temporal gyri, inferior and superior parietal lobule (BA 7/40), pre- and postcentral gyri, lateral ACC (BA 32), bilateral PCC (BA 29/30), PHIPP, putamen, thalamus, tonsil, declive, pyramis, culmen and pons in the brainstem area. On the other hand, the network of the R1 period includes the bilateral frontal gyri (BA 10/11), STG (BA 39), lateral postcentral gyrus, precuneus, declive and pyramis.

However, during the second needle manipulation and resting states, the ICA results (Fig. 2) indicated that they have almost the same functional network. It includes the bilateral frontal gyri, bilateral temporal gyri, IPL, the middle occipital gyri (MOG), pre- and postcentral gyri, ACC, PHIPP, insula, tonsil, pyramis, culmen, precuneus and cuneus. During the S2 state, the network also has the lateral PCC, caudate, putamen, and thalamus, while the network of $\mathrm{R} 2$ includes the superior parietal lobule (SPL) and pons.

The ICA method can be used to identify spatially independent sources from linearly mixed fMRI data [23]. The activated regions identified in one component were driven via a similar time course and constituted a specific functional network [6]. The current results of ICA demonstrated the functional networks' diversity as seen between RSBNS and the R1 and R2 states, and it also indicated that there existed different functional networks between the 'on-state' and 'off-state.'

The result of the RSBNS state network, which is located in the frontal and temporal gyri, was consistent with a ventral/reorienting attention network [8], the SPL as well as the precuneus, both known to be active during spatial attention [18], while the pre- and postcentral gyri, ACC, and HIPP corresponded to the default mode network (DMN) [25]. The result of these activated areas is consistent with the resting-state networks (RSNs) [26]. During the R1 period, the functional network only included the frontal gyri, lateral STG, 


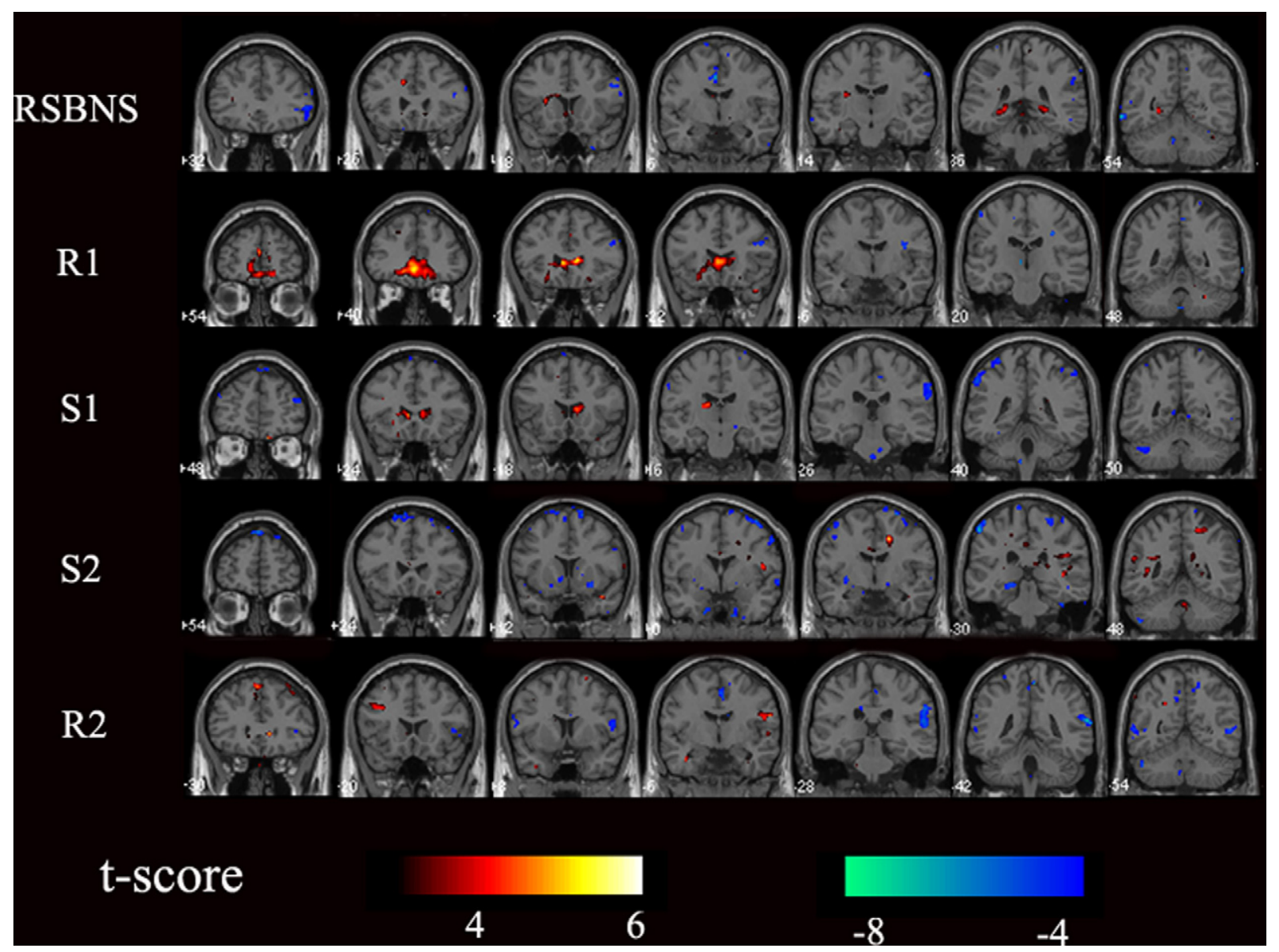

Fig. 2. The functional networks of RSBNS, R1, S1, S2 and R2 states. (For interpretation of the references to color in this artwork, the reader is referred to the web version of the article.)

precuneus, lateral postcentral gyrus, ACC (BA 24/32), declive and pyramis in the cerebellum. Compared to the RSBNS, the coactivation areas of the ACC and frontal gyri are more extensive during R1. The rostal part of the ACC, which is connected to the amygdala and the periaqueductal gray matter (PAG), modulates the internal emotional response to pain [9]. The frontal gyri are consistent with activations identified in previous human experimental neuroimaging studies of pain [10]. The different functional networks indicated that the baseline changed after the needle manipulation. During the 'off-state,' the remaining expectation and acupuncture effects as well as some of the lasting sensation to the needle stimulation modulated the functional brain network.

The functional network extracted from the S1 period is more complicated than the one in R1. Although they have the same area located in the frontal, temporal and parietal gyri, ACC, precuneus and postcentral gyrus, $S 1$ still included the inferior and middle temporal gyri, precentral gyrus, PCC (BA 29/30), PHIPP, putamen, thalamus, tonsil, and culmen. The temporal gyri activation is known to be modulated by pain and noxious insults [1]. The putamen is known to be crucial for motor activity [24]. The thalamus was identified in pain studies [10]. The postcentral is the SII area as well as the PHIPP, tonsil, and culmen in the cerebellum, which are involved in processing incoming sensory stimulation and high pain sensation of the subjects [10]. These different activation areas of the networks were critical in the analysis regarding the 'on-state' and 'off-state.'

The discrepancy between R2 and S2 is less than that of R1 and S1. The functional network of R2 resembles S2. They both contain the most number of identical areas consisting of the bilateral frontal gyri, bilateral temporal gyri, IPL, MOG, pre- and postcentral gyri, ACC, PHIPP, insula, tonsil, pyramis, culmen, precuneus and cuneus. Only the PCC, caudate, putamen, and thalamus are still active in S2. Based on our analysis, we thought there existed different effects between the 'on-state' and 'off-state,' in which subjects already experienced two needle stimulation blocks. We predicted that there still remains a lasting sensation to the mechanical stimulation except for expectation and acupuncture effects. Therefore, as the stimulation proceeds, we infer that the effects between the 'on-state' and 'off-state' are closer than before, and when acupuncture ceases, the effects can last for hours or days according to TCM theories.

Using the ICA method, we discovered a variety of resting state networks before and after needle manipulation, and the continuous changing of the resting state functional network during the experimental paradigm. The results indicated that real acupuncture effects are not being altered due to the multiple-block paradigm, because the resting state after needle stimulation did not return to the RSBNS state. Furthermore, the discrepancy between S1 and R1, and S2 and R2 showed that there exist different functional brain networks between the 'on-state' and 'off-state.' Instead, the networks of these two states are changing as the stimulation paradigm continues.

\section{Acknowledgments}

This paper is supported by the Project for the National Key Basic Research and Development Program (973) under Grant No. 2006CB705700, Changjiang Scholars and Innovative Research Team 
in University PCSIRT under Grant No. IRT0645, CAS Hundred Talents Program, CAS scientific research equipment develop program (YZ0642, YZ200766), 863 program under Grant No. 2006AA04Z216, 2008AA01Z411, the Joint Research Fund for Overseas Chinese Young Scholars under Grant No. 30528027, the National Natural Science Foundation of China under Grant Nos. 30672690, 30600151, 30500131, 30873462, 30870685, 60532050, 60621001, 90809002, Beijing Natural Science Fund under Grant No. 4071003.

\section{References}

[1] L.R. Becerra, H.C. Breiter, M. Stojanovic, S. Fishman, A. Edwards, Human brain activation under controlled thermal stimulation and habituation to noxious heat: an fMRI study, Magnetic Resonance in Medicine 41 (1999) 1041-1057.

[2] C.F. Beckmann, S.M. Smith, Probabilistic independent component analysis for functional magnetic resonance imaging, IEEE Transactions on Medical Imaging 23 (2004) 137-152.

[3] A.J. Bell, T.J. Sejnowski, An information-maximization approach to blind separation and blind deconvolution, Neural Computation 7 (1995) 1129-1159.

[4] B. Biswal, E. DeYoe, J. Hyde, Reduction of physiological fluctuation in fMRI using digital filters, Magnetic Resonance in Medicine 35 (1996) 107-113.

[5] V.D. Calhoun, T. Adali, G.D. Pearlson, J.J. Pekar, A method for making group inferences from functional MRI data using independent component analysis, Human Brain Mapping 14 (2001) 140-151.

[6] V.D. Calhoun, J.J. Pekar, V.B. McGinty, Different activation dynamics in multiple neural systems during simulated driving, Human Brain Mapping 16 (2002) 158-167.

[7] Z.H. Cho, S.C. Chung, J.P. Jones, J.B. Park, H.J. Lee, E.K. Wong, B.I. Min, New findings of the correlation between acupoints and corresponding brain cortices using functional MRI, Proceedings of the National Academy of Sciences of the United States of America 95 (1998) 2670-2673.

[8] M. Corbetta, G.L. Shulman, Control of goal-directed and stimulus-driven attention in the brain, Nature Reviews Neuroscience 3 (2002) 201-215.

[9] O. Devineky, M.J. Morrell, B.A. Vogt, Contributions of anterior cingulate cortex to behavior, Brain 118 (1995) 279-306.

[10] S.W. Derbyshire, A.R. Jones, F. Gyulai, S. Clark, D. Townsend, L.L. Firestone, Pain processing during three levels of noxious stimulation produces differential patterns of central activity, Pain 73 (1997) 431-445.

[11] D. Diehl, G. Kaplan, I. Coulter, D. Glik, E.L. Hurwitz, Use of acupuncture by American physician, Journal of Alternative and Complementary Medicine 3 (1997) $119-126$.

[12] J.R. Duann, T.P. Jung, W.J. Kuo, Single-trial variability in event-related BOLD signals, NeuroImage 15 (2002) 823-835.

[13] D.M. Eisenberg, R.C. Kessler, C. Foster, Unconventional medicine in the United States, New England Journal of Medicine 328 (1993) 246-252.

[14] D.M. Eisenberg, R.B. Davis, S.L. Ettner, S. Appel, S. Wilkey, M. Van Rompay, R.C. Kessler, Trends in alternative medicine use in the United States, Journal of the American Medical Association 280 (1997) 1569-1575.

[15] K.J. Friston, J. Ashburner, C.D. Frith, J.B. Poline, J.D. Heather, R.S.D. Frackowiak, Spatial registration and normalization of images, Human Brain Mapping 2 (1995) 165-189.
[16] K.J. Friston, C.D. Frith, P.F Liddle, R.S. Frackowiak, Comparing functional (PET) images: the assessment of significant change, Journal of Cerebral Blood Flow and Metabolism 11 (1991) 690-699.

[17] K.J. Friston, S. Williams, R. Howard, R.S. Frackowiak, R. Turner, Movementrelated effects in fMRI time-series, Magnetic Resonance in Medicine 35 (1996) 346-355.

[18] C. Grefkes, G.R. Fink, The functional organization of the intraparietal sulcus in humans and monkeys, Journal of Anatomy 1 (2005) 3-17.

[19] M.D. Greicius, G. Srivastava, A.L. Reiss, V. Menon, Default-mode network activity distinguishes Alzheimer's disease from healthy aging: evidence from functional MRI, Proceedings of the National Academy of Sciences of the United States of America 101 (2004) 4637-4642.

[20] K.K. Hui, J. Liu, O. Marina, V. Napadow, C. Haselgrove, K.K. Kwong, D.N. Kennedy, N. Markris, The integrated response of the human cerebro-cerebellar and limbic systems to acupuncture stimulation at ST 36 as evidenced by fMRI, Neuroimage 27 (2005) 479-496.

[21] J. Kong, L. Ma, R.L. Gollub, J. Wei, A pilot study of functional magnetic resonance imaging of the brain during manual and electroacupuncture stimulation of acupuncture point (LI-4 Hegu) in normal subjects reveals differential brain activation between methods, Journal of Alternative and Complementary Medicine 8 (2002) 411-419.

[22] M.J. McKeown, T.P. Jung, Spatially independent activity patterns in functional MRI data during the Stroop color-naming task, Proceedings of the National Academy of Sciences of the United States of America 95 (1998) 803810.

[23] M.J. Mckeown, S. Makeig, G.G. Brown, T.P. Jung, S.S. Kindermann, A.J. Bell, T.J. Sejnowski, Analysis of fMRI data by blind separation into independent spatial components, Human Brain Mapping 6 (1998) 160-188.

[24] B.V. Manyam, J.R. Sancher-Ramos, Traditional and complementary therapies in Parkinson's disease, Advances in Neurology 80 (1999) 565-574.

[25] M.E. Raichle, A.M. MacLeod, A.Z. Snyder, W.J. Powers, D.A. Gusnard, G.L. Shulman, A default mode of brain function, Proceedings of the National Academy of Sciences of the United States of America 2 (2001) 676-682.

[26] C. Sorg, V. Riedl, M. Mühlau, V.D. Calhoun, T. Eichele, L. Läer, A. Drzezga, H. Förstl, A. Kurz, C. Zimmer, A.M. Wohlschläger, Selective changes of resting-state networks in individuals at risk for Alzheimer's disease, Proceedings of the National Academy of Sciences of the United States of America 104 (2007) 1876018765.

[27] R.M. Weisskoff, Simple measurement of scanner stability for functional NMR imaging of activation in the brain, Magnetic Resonance in Medicine 36 (1996) 643-645.

[28] M.T. Wu, J.C. Hsieh, J. Xiong, C.F. Yang, H.B. Pan, Y.C.I. Chen, G.C. Tsai, B.R. Rosen, K.K. Kwong, Central nervous pathway for acupuncture stimulation: localization of processing with functional MR imaging of the brain-preliminary experience, Radiology 212 (1999) 133-141.

[29] M.T. Wu, J.M. Sheen, K.H. Chuang, P. Yang, S.L. Chin, C.Y. Tsai, C.J. Chen, J.R. Liao, P.H. Lai, K.A. Chu, H.B. Pan, C.F. Yang, Neuronal specificity of acupuncture response: an fMRI study with electroacupuncture, Neuroimage 16 (2002) 1028-1037.

[30] B. Yan, K. Li, J.Y. Xu, W. Wang, Acupoint-specific fMRI patterns in human brain, Neuroscience Letters 383 (2005) 236-240.

[31] W.T. Zhang, Z. Jin, F. Luo, L. Zhang, Y.W. Zeng, J.S. Han, Evidence from brain imaging with fMRI supporting functional specificity of acupoints in humans, Neuroscience Letters 354 (2004) 50-53. 